\title{
Structural heterogeneities and electronic effects in self-organized core-shell type structures of $\mathbf{S b}$
}

\author{
T. V. Kulikova ${ }^{1, \dagger}$, L. A. Bityutskaya ${ }^{1}$, A. V. Tuchin¹, E. V. Lisov², S. I. Nesterov², \\ A. A. Averin ${ }^{3}$, B. L. Agapov ${ }^{4}$ \\ †kaimt@mail.ru \\ ${ }^{1}$ Voronezh State University, University Square, 1, Voronezh, 394018, Russia \\ ${ }^{2}$ NT-MDT Spectrum Instruments, Zelenograd transit № 4922, 4, Moscow, 124460, Russia
}

\begin{abstract}
${ }^{3}$ Institute of Physical Chemistry and Electrochemistry of RAS, Leninsky Prospekt, 31, Moscow, 119071, Russia
${ }^{4}$ Center for collective use of scientific equipment, Voronezh State University, University Square, 1, Voronezh, 394018, Russia

The paper provides morphological and electro-physical characteristics of the set of structures of the core-shell type of antimony ranging from $10^{-4}$ to $10^{-6} \mathrm{~m}$, obtained in a single-stage process as a result of spontaneous crystallization of the melt. It verifies that the structures obtained can be considered as an example of the new type of core-shell structures in a series of self-organized structures derived from their layered precursor. The structures consist of different forms of the same substance, where the core is represented by mono-crystalline gray antimony and the shell is a deformed two-dimensional film. Based on the overall data obtained, the shell of the structure can be described as a cover film with a variable thickness, which contains structural heterogeneities in the form of antimony allotropes, i.e. defective antimonene nano-layers with a high proportion of boundary atoms and dangling bonds. Structural heterogeneity foster electronic effects such as: localized charge contrast, which occurs when an electron beam is applied; emerging of conductive and non-conducting areas on the surface of the shell; electrostatic interaction of particles; ability of the structures to accumulate an excess charge and retain it for a long time. The change in the properties of the nano-shell of the spheroidal structure of the core-shell type of antimony can be considered as a consequence of its deformed structure.
\end{abstract}

Keywords: antimony, antimonene, core-shell structure, deformation, charge contrast, allotrope.

\section{Introduction}

Since the self-organized hollow carbon nanostructures, such as fullerenes and graphite nano-tubes were discovered, much attention has been paid to get non-carbon nanostructures from other layered materials [1-4]. Hollow and solid spherical nanostructures of various compounds with a core-shell structure are allocated in a separate group. Core-shell structures are highly functional materials with modified properties that can be changed by replacing constituent materials or the core-to-shell ratio. Due to the high variability of properties, the core-shell type structures have a wide range of possible applications at the junction of different areas [5-9].

Along with such materials as dichalcogenides, $\mathrm{A}^{\mathrm{III}} \mathrm{B}^{\mathrm{VI}}$ semiconductors, halogen-containing materials, etc., the elements of group $\mathrm{V}$ and their compounds have a pronounced layer structure [10-12].

Currently, graphene-like structures formed by the elements of the $\mathrm{V}$ group are distinguished as a separate group. In overall scientific literature, the names of layered allotropes are already confirmed: the arsenene - the monolayer of gray arsenic $[13,14]$, the phosphorene - the monolayer of black phosphorus [15-17], and the antimonene - the monolayer of gray antimony [18]. From both - scientific and practical point of view - the greatest interest is represented by antimony as a material with the most stable structure.

Unlike graphene, antimonene demonstrates the presence of a band gap of 0.2 to $2.28 \mathrm{eV}$, depending on configuration of the layers, which creates high potential for its use in electronics and optoelectronics [19-22]. In addition, antimony, as a heavy element, which can serve as a pattern for investigating topological effects, depending on the thickness of the layer of crystalline material [23-26].

It is theoretically verified that antimonene is sensitive to deformations of the layer, which influence the formation of topological phases. The paper [27] shows the change in the electronic structure of antimonene affected by deformations and its transition to the state of topological insulator (TI) at the critical value of tensile stresses.

Moreover, a similar response of the $2 \mathrm{D}$ allotrope to the distortion of the structure is characteristic not only of antimony, but of other pnictides and its compounds [28, 29]. The antimonene bilayer also demonstrates the change in the electronic structure as well as in the formation of the quantum spin and anomalous Hall phase during deposition of atomic hydrogen, fluorine or nitrogen [30].

At present, the topological context of the properties of elementary $2 \mathrm{D}$ materials is undergoing its initial stage of research. It requires development of the new theoretical and 
experimental approaches, which should consider the specific interlayer interaction in the original crystalline materials.

It remains crucial to obtain stable modifications of $2 \mathrm{D}$ materials with reproducible properties and to distinguish them, as well as to create composite materials that enhance the stability of layered modifications and expand the range of their functional properties.

In the papers $[31,32]$ an array of core-shell structures was first obtained from a layered precursor with a strong interlayer interaction - antimony via its self-formation from the melt.

This paper studies structural features and properties of self-organized structures of the core-shell type antimony obtained from the melt.

\section{Characteristics of the object under research}

Spheroidal structures of $\mathrm{Sb}$, obtained as a result of spontaneous crystallization of the melt could be used as the subject matter, provided the following conditions are met:

- The weight of the initial antimony sample does not exceed $3 \mathrm{~g}$ and depends on the size of the particular vessel. It has $99.999 \%$ purity;

- Vacuumed $10^{-4} \mathrm{~Pa}$ quartz vessel of special shape coaxially connected cylindrical quartz vessels of 14 and $7 \mathrm{~mm}$. The inner vessel is open and used to control the temperature in the reactor:

- Average cooling rates of the melt are $1-100 \mathrm{~K} /$ second.

As the melt cools down and all the above conditions are met, spontaneous granulation of the melt is observed, which leads to the formation of an array of unbound spheroidal antimony particles in the ranging from $10^{-4}$ to $10^{-6} \mathrm{~m}$.

The array was fractionated into different sizes using standard laboratory sieves. The number of particles in the array obtained during one technological cycle is about 17000 , whereas more than $50 \%$ of particles are $10^{-6} \mathrm{~m}$ size.

The antimony particles obtained as a result of spontaneous crystallization are stable chemically, morphologically and functionally if stored for a long-term in the open air conditions and do not form agglomerates.

The particles easily break off along the cleavage plane of antimony (0001), forming a single-crystal flattish cleavage. As a result of diffraction studies, the increase in parameters and volume of the unit cell and the loosening of the structure of Sb spheroidal particles [31] were observed.

On the surface of spheroidal particles of antimony an insignificant amount of locally concentrated of impurities of oxygen and carbon were detected (Fig. 1). Local concentration areas of the impurity in the volume of spheroidal particles were not detected.

If studied visually and macroscopically, the electrostatic interaction of individual antimony particles in the form of repulsion of particles when moving close to each other is observed, although they show no interaction during the storage, since electrostatic equilibrium is being established at this stage.

The most intense repulsion of particles appears as we try to grind the particles mechanically. Separate particles also interact with polymer and metal substances and preserve an excess charge for a long time.

\section{Research method}

Since the subject is still poorly studied the research on spheroidal antimony particles was carried out as a complex of complementary, experimental and theoretical methods:

- Scanning electron microscopy (SEM) of spheroidal $\mathrm{Sb}$ particles ranging from $10^{-4}$ to $10^{-6} \mathrm{~m}$ was carried out with Jeol JSM-6380LV microscope; Element analysis by INCA Energy 250 microanalysis system;

- Raman spectroscopy was carried out with Senterra "Bruker" spectrometer. To register the Raman spectra of spheroidal $\mathrm{Sb}$ particles ranging from $10^{-4}$ to $10^{-6} \mathrm{~m}$, a laser $\lambda=532 \mathrm{~nm}$ was used as the radiation source. The spectra were obtained under the following conditions: the $20 \times$ objective, the spot diameter was $3 \mu \mathrm{m}$, the laser radiation power ranging from 0.2 to $20 \mathrm{~mW}$, the signal accumulation of 100 units at the room temperature. Crystalline parent antimony was used as a reference;

- The quantum-chemical modeling of Raman spectra was carried out based on the density functional theory (DFT) using the Gaussian09 software complex at the "Supercomputer Center" of Voronezh State University. The subjects of the study were the fragments of graphene and $5 \times 5$ antimonene elementary cells passivated with hydrogen at their ends. Antimony contained six-membered rings, where the distances between the atoms comprised $2.88 \AA$, which corresponds to the rhombohedral modification of $\mathrm{R} \overline{3} \mathrm{~m}$ antimony;

- $\quad$ Atomic Force Microscopy (AFM) was carried out with Solver Next microscope manufactured by NT-MDT Spectrum Instruments with additional hybrid controller. NSG10 cantilever coated with platinum. Spheroidal Sb particles fractions of $350 \mu \mathrm{m}$.

\section{Main results}

As the SEM method was applied with a change in the magnification ranging from of 5000 to $200000 \times$, the presence of a non-continuous film of variable thickness forming the shell of the spheroidal particle was discovered in all the parts of the surface of the spheroidal particle (Fig. 2). The morphology of the film shows the wrinkled surface typical for graphene sheets. At the study of the film by SEM method in the regime of secondary electrons with magnification of $100000 \times$ was recorded that the film on the surface of the spheroidal particle semitransparent for the electrons.

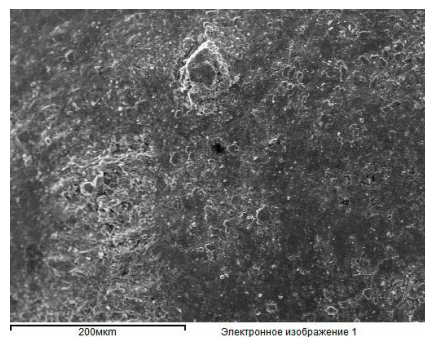

a

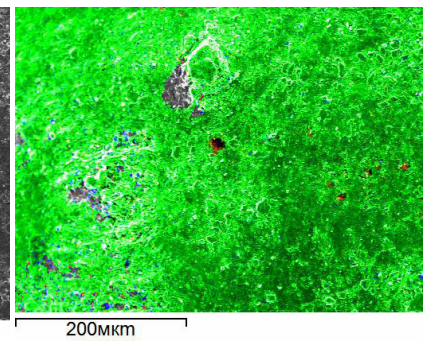

b
Fig. 1. (Color online) SEM image of the surface of spheroidal particles and elements distribution map on the surface. Antimony is marked in green and carbon in red. 
Estimating the size of the interaction area of the electron beam with the sample based on Kanaye and Okayama [33], and taking into account the maximum depth of the yield of secondary electrons, the smallest film thickness is about $2 \mathrm{~nm}$.

The phase micro-heterogeneities of the film are detected - single flat crystallites (Fig. 3). The analogous antimony sheets were obtained in the process of Van der Waals epitaxy [34] and were interpreted by the authors as 2D allotrope of antimony - antimonene.

Analysis of the deformed surface of spheroidal antimony particles was carried out using the Raman spectroscopy method. Based on the Raman spectra of the surface of spheroidal $\mathrm{Sb}$ particles, regardless of the fraction, the vibration modes typical for crystalline antimony - with the peaks of 115 and $152 \mathrm{~cm}^{-1}$ - are observed, along with intense diffuse peaks in the high-frequency area of 1344 and $1604 \mathrm{~cm}^{-1}$ (Fig. 4).

The Raman spectrum of the volume of spheroidal particles does not contain high-frequency features and coincides with the spectrum of the reference crystalline material.

In some of the papers [35-37], when analyzing the Raman spectra of antimony-carbon composites, highfrequency modes in the $1300-1600 \mathrm{~cm}^{-1}$ area are represented as $D$ and $G$ modes that are typical for graphite-like film on the sample surface. However, under the conditions of synthesis of spheroidal particles of antimony, the formation of a graphite-like film is unlikely, which is confirmed by the results of a microanalysis, where it is shown that carbon does

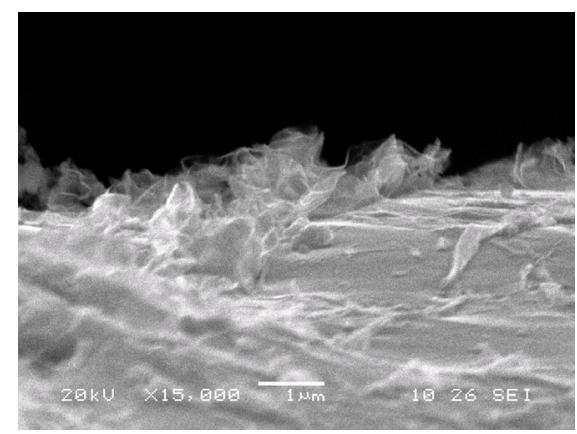

Fig. 2. Film on the surface of spheroidal particles of antimony, $15000 \times, 20 \mathrm{kV}$.

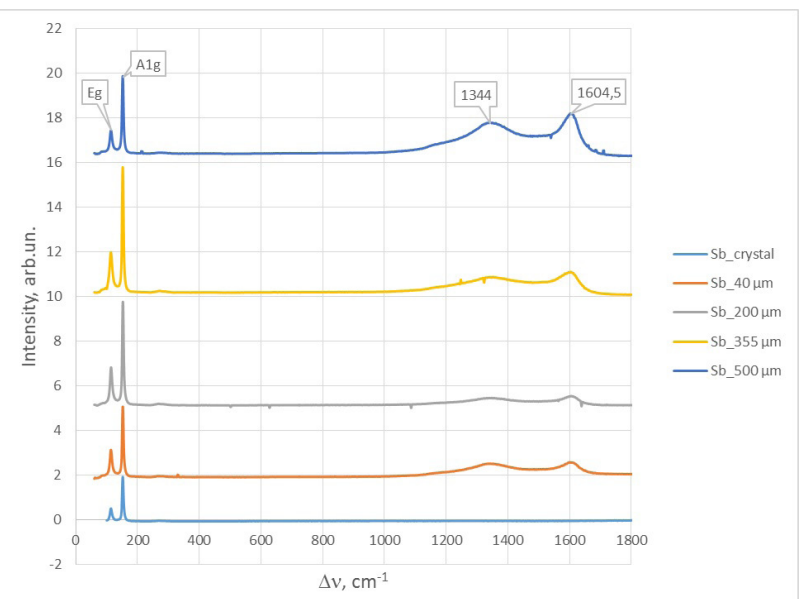

Fig. 4. (Color online) The Raman spectra of spheroidal particles of antimony and of the reference material. not cover the surface of spheroidal particles everywhere as a film, but is concentrated in local nanoregions (Fig. 1). Therefore, the overall analysis of high-frequency peaks is difficult.

In order to identify the nature of the experimental highfrequency doublet, quantum-chemical modeling of the Raman spectra of fragments of graphene and antimonene layers was carried out.

In order to take into account the heterogeneity and defectiveness of the deformed shell that was detected experimentally (Fig. 2, 3), the fragments of antimonene and graphene $5 \times 5$ layers measuring unit cells, which were passivated by hydrogen at their ends was used as a model of the theoretical investigation in the present study. For a fragment of the graphene layer, a series of additional peaks appear in the D- and G-mode areas, determined by the vibrations of the atoms of the layer boundaries, the most intense modes are observed in the areas of $1200,1400,1620 \mathrm{~cm}^{-1}$ and $1690 \mathrm{~cm}^{-1}$ (Fig. 5a).

The spectra of the fragment of the antimony film contain antimony modes in the area of up to $200 \mathrm{~cm}^{-1}$ size, as well as in bulk samples. In addition, a series of modes were observed in the area of $1305 \mathrm{~cm}^{-1}$ and $1640 \mathrm{~cm}^{-1}$, which exceed the antimony mode intensity by 3 to 10 units, corresponding to the vibrations of the bonds of antimony and hydrogen atoms at the film boundary (Fig. 5b).

Thus, experimentally detected modes on the surface of spheroidal antimony particles can be referred to as modes of defective antimonene layers with high proportion of

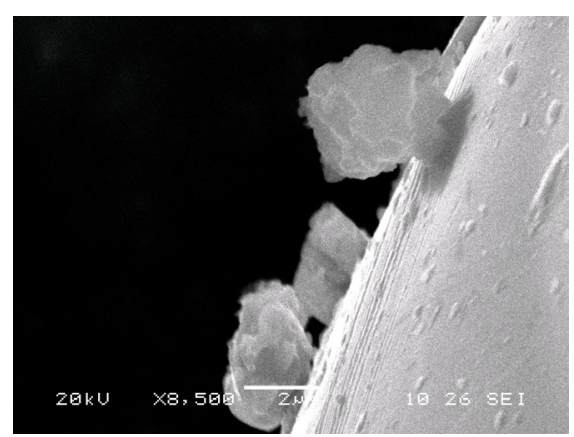

Fig. 3. Typical elements of the surface morphology of spheroidal particles of antimony: flat crystallites, $8500 \times, 20 \mathrm{kV}$.
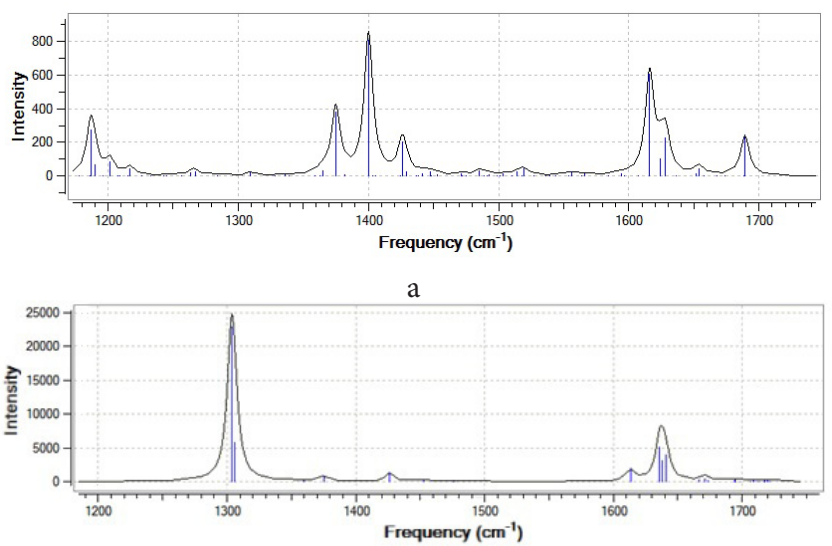

b

Fig. 5. Calculated Raman spectra of the graphene fragment (a) and antimonene fragment (b). 
boundary atoms and dangling bonds, whereas the shell is considered as a multiphase multilayer structure of the antimony allotrope. By estimating the changes in the relative intensities of the peaks Eg and A1g based on the method proposed in the paper [20], the largest thickness of the multiantimony on the surface of the antimony spheroidal structure is about $19.5 \mathrm{~nm}$.

In the theoretical studies conducted for the model of an infinite not deformed antimony sheet high-frequency peaks are not observed [38].

The film is thermally stable under normal conditions, but it destructed with increasing the temperature due to increasing laser radiation power of up to $20 \mathrm{~mW}$, which is verified by the absence of the high-frequency doublet and the appearance of modes typical for antimony oxides.

Studying the properties of an array of core-shell structures based on antimony under normal conditions shows the unusual effect performed by the conductive material.

During the SEM measurements with magnification of the image resolution of the surface of antimony particles reaching $200000 \times$, a localized charge contrast is detected in the secondary electron mode. Thus, the film on the surface of antimony particles is no longer "semi-metallic", but exhibits dielectric properties.

During the research of the surface of spheroidal antimony particles, the AFM method confirmed the developed morphology of the particle surface and detected its dielectric properties. In the spreading current regime at a voltage of $1 \mathrm{~V}$, the topological heterogeneity of the conductivity is detected, which is verified by the set of conducting and nonconducting areas (Fig. 6). Moreover, the conducting areas are much smaller and could be caused by elastic stresses in the structure or by mechanical damages of the shell. Thin dielectric surface layer partially explains the visually observed electrostatic effects.

\section{Discussion of the results}

Currently, a huge number of core-shell structures has been obtained. The main features of such structures are:

- various core/shell materials;

- the overall goal is to form a defect-free boundary;

- multi-stage sequential build-up or self-organization in high-energy flows.

Spheroidal antimony particles can be considered as a representative of a new type of core-shell structures in a

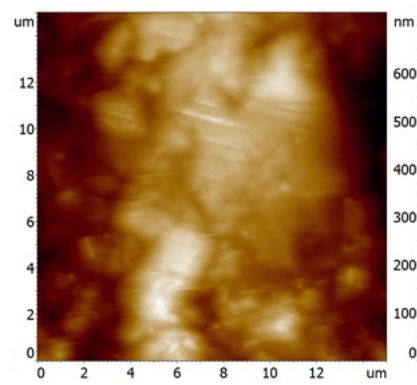

a

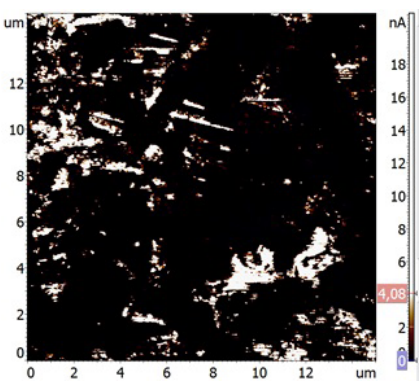

b
Fig. 6. AFM image of the surface relief (a) and current flow (b) of the spheroidal particle of antimony. series of self-organized structures deriving from a layered precursor. Structures of the core-shell type of antimony consist of different forms of the same substance: the core is represented by the single-crystal gray antimony, and the shell by the deformed 2D film.

Based on the overall morphological and spectral data obtained, the shell of the antimony spheroidal structure can be regarded as a quasi-independent cover film of variable thickness containing structural heterogeneities. Its structure is similar to the natural structures of the core-shell, such as nut, for example, which core is situated in some sort of a shell with the properties different of those of the nut itself.

The formation of structures of this type on one hand derives from the antimony's polymorphism tendency, on the other, from its propensity to form "metastable phases", including amorphous ones. This ability is determined by the nonequivalence of the bonds in the crystal structure of the material, which is due to the different values of the typical temperatures and frequencies [39].

Antimony atoms lie in two sheets and, thus, form some sort of a corrugated structure of layers, which predetermines the sensitivity of the structure to external influences - size effects and deformations [18].

As far as flat non-deformed structures are concerned, topological effects appear as dimensional ones. According to theoretical calculations, as the dimensionality decreases from $3 \mathrm{D}$ to $2 \mathrm{D}$ material, a number of topo-electronic transitions occur in crystalline antimony: from the $3 \mathrm{D}$ topological semimetal to the $3 \mathrm{D}$ topological insulator (TI) with the thickness of $7.8 \mathrm{~nm}$, which corresponds to 22 bilayers of the material, then to $2 \mathrm{D}$ TI at $2,7 \mathrm{~nm}$ ( 8 bilayers) and to a trivial semiconductor at $1 \mathrm{~nm}$ (3 bilayers) [23].

Topological effects deriving from the deformation process of the allotropic structure occur under the influence of external influences. As it was demonstrated in the papers [38, 40] the starter mechanism of induced spin states in antimony differs significantly from other 2D materials: band inversion is not related to the elongation of covalent bonds but to the change in the bond angles.

Therefore, the promising perspective of such research is to study the properties of the non-planar deformed structure of antimonene and multi-antimonene.

The impact of deformations on the properties of a nanoshell of a structure of the core-shell type of antimony is represented in this paper by means of the following effects:

- localized charge contrast arising when the electron beam is applied;

- conductive and non-conducting areas on the surface of the shell;

- electrostatic interaction of particles;

- ability to accumulate excess charge and save it for a long time.

Thus, the results of our work can be considered as an experimental verification of the possibility to control the properties of antimony allotropes by deformation effects predicted by the papers $[27,38,40]$.

Acknowledgements. We express our gratitude to the Center for collective use of scientific equipment of the Voronezh State University for active support of the work of young scientists. 


\section{References}

1. R. Tenne, R. Rosentsveig, A. Zak. Phys. Status Solidi A. 210 (11), 2253 - 2258 (2013), DOI: 10.1002/pssa.201329309

2. C.N. R. Rao, M. Nath. Dalton Transactions. 1, 1-24 (2003), DOI: 10.1039/B208990B

3. G. Compagnini, M. G. Sinatra, G. C. Messina, G. Patane, S. Scalese, O. Puglisi. Applied Surface Science. 258 (15), 5672 - 5676 (2012), DOI:10.1016/j.apsusc.2012.02.053

4. R. Tenne. Frontiers of Physics 9 (3), 370-377 (2014), DOI:10.1007/s11467-013-0326-8

5. B. Kalska-Szostko, U. Wykowska, D. Satuła. Colloids and Surfaces A: Physicochemical and Engineering Aspects. 481, 527 - 536(2015), DOI: 10.1016/j.colsurfa.2015.05.040

6. A.V. Nomoev, S.P. Bardakhanov, M. Schreibe, D. G. Bazarova, N.A. Romanov, B.B. Baldanov, B. R. Radnaev, V. V. Syzrantsev. Beilstein J. Nanotechnol. 6, 874 - 880 (2015), DOI:10.3762/bjnano.6.89

7. F. Weis, M. Seipenbusch, G. Kasper, Film Growth. Materials 8 (3), $\quad 966-976$ (2015), DOI:10.3390/ ma8030966

8. H. J. Choi, W.L. Zhang, S. Kim, Y.Seo. Materials 7 (11), 7460 - 7471 (2014), DOI:10.3390/ma7117460

9. R. G. Chaudhuri, S. Paria. Chem. Rev. 112 (4), 2373-2433 (2012), DOI: 10.1021/cr100449n

10. N.C. Norman. Chemistry of Arsenic, Antimony and Bismuth. Springer Science+Business Media B. V., Springer Netherlands. (1998) 484 p.

11. J. Donohue. The Structures of the Elements. John Wiley, New York. (1974) 436 p.

12. J. J. Zuckerman, A.P. Hagen. Inorganic Reactions and Methods, The Formation of Bonds to N, P, As, Sb, Bi. WILEY-VCH. (1988) 385 p.

13. C. Kamal, Motohiko Ezawa. Phys. Rev. B. 91, 085423 (2015), DOI: 10.1103/PhysRevB.91.085423

14. W. Xu, P. Lu, L. Wu, C. Yang, Y. Song, P. Guan, L. Han, S. Wang. IEEE Journal of Selected Topics in Quantum Electronics. 23 (1), 9000305 (2017), DOI: 10.1109/ JSTQE.2016.2593106

15. A. Carvalho, M. Wang, X. Zhu, A.S. Rodin, H. Su, A.H. Castro Neto.Nature Reviews Materials. 1, 16061 (2016), DOI:10.1038/natrevmats.2016.61

16. A. Castellanos-Gomez, L. Vicarelli, E. Prada, J. O. Island, K. L. Narasimha-Acharya, S. I. Blanter, D. J. Groenendijk, M. Buscema, G. A. Steele, J. V. Alvarez, H. W. Zandbergen, J. J. Palacios, H.S. J van der Zant. 2D Materials. 1, 025001 (2014), DOI:10.1088/2053-1583/1/2/025001

17. S. Bagheri, N. Mansouri, E. Aghaie. International Journal of Hydrogen Energy. 41 (7), 4085 - 4095 (2016), DOI:10.1016/j.ijhydene.2016.01.034

18. O. Uzengi Akturk, V. Ongun Ozcelik, S. Ciraci. Physical Review B. 91, 235446 (2015), DOI: 10.1103/ PhysRevB.91.235446

19. Y. Xu, B. Peng, H. Zhang, H. Shao, R. Zhang, H. Lu, D. Wei Zhang, H. Zhu. (2016), arXiv:1604.03422

20. C. Huo, X. Sun, Z. Yan, X. Song, S. Zhang, Z. Xie, J. Liu, J. Ji, L. Jiang, S. Zhou, H. Zeng. J. Am. Chem. Soc. 139 (9), 3568 - 3568 (2017), DOI: 10.1021/jacs.6b08698

21. P. Ares, F. Aguilar-Galindo, D. Rodríguez-San-Miguel, D. A. Aldave, S. Díaz-Tendero, M. Alcamí, F. Martín,
J. Gómez-Herrero, F. Zamora. Adv. Mater. 28 (30), 6332 - 6336 (2016), DOI: 10.1002/adma.201602128

22. S. Zhang, Z. Yan, Y. Li, Z. Chen, H. Zeng. Angew. Chem.Int. Ed. 54, 1-5 (2015), DOI: 10.1002/ anie. 201411246

23. P. Zhang, Z. Liu, W. Duan, F. Liu, J.Wu. Physical Review B. 85, 201410 (R) (2012), DOI: 10.1103/ PhysRevB.85.201410

24. G. Bian, T. Miller, T.-C. Chiang. Physical Review Letters PRL. 107, 036802 (2011), DOI: 10.1103/ PhysRevLett.107.036802

25. S. H. Kim, K-H. Jin, J. Park, J. S. Kim, S-H. Jhi, H. W. Yeom. Scientific Reports. 6, 33193 (2016), DOI: 10.1038/ srep33193

26. G. Yao, Z. Luo, F. Pan, W. Xu, Y.P. Feng, X-S. Wang. Scientific Reports. 3, 2010 (2013), DOI: 10.1038/ srep02010

27. J. Liang, L. Cheng, J. Zhang, H. Liu. (2015), arXiv:1502.01610

28. Y. Nie, M. Rahman, D. Wang, C. Wang, G. Guo. Scientific Reports. 5, 17980 (2015), DOI: 10.1038/srep 17980

29. S. Zhang, M. Xie, B. Cai, H. Zhang, Y. Ma, Z. Chen, Z. Zhu, Z. Hu, H. Zeng. Physical Review B. 93, 245303 (2016), DOI: 10.1103/PhysRevB.93.245303

30. K.-H. Jin, S.-H. Jhi. Scientific Reports. 5, 8426 (2015), DOI: $10.1038 /$ srep08426

31. T. V. Kulikova, L.A. Bityutskaya, A.V. Tuchin, A. A. Averin. Journal of Advanced Materials. 3, 5-13 (2017). (in Russian) [Т. В. Куликова, Л.А. Битюцкая, А. В. Тучин, А. А. Аверин. Перспективные материалы. 3, $5-13$ (2017)]

32. T. V. Kulikova, L.A. Bityutskaya. Condensed Matter and Interphases 18 (1), 61-66 (2016). (in Russian) [Т.В. Куликова, Л. А. Битюцкая. Конденсированные среды и межфазные границы. 18 (1), 61 - 66 (2016)]

33. J. Goldstein, D.E. Newbury, D.C. Joy, C.E. Lyman, P. Echlin, E. Lifshin, L. Sawyer, J. R. Michael. Scanning Electron Microscopy and X-ray Microanalysis. Springer US, New York. (2003) 689 p.

34. J. Ji, X. Song, J. Liu, Z. Yan, C. Huo, S. Zhang, M. Su, L. Liao, W. Wang, Z. Ni, Y. Hao, H. Zeng. Nature Communications. 7, 13352 (2016), DOI: 10.1038/ncomms 13352

35. N. Zhang, Y. Liu, Y. Lu, X. Han, F. Cheng, J. Chen. Nano Research. 8 (10), $3384-3393$ (2015), DOI: 10.1007/ s12274-015-0838-3

36. T. Ramireddy, Md. Mokhlesur Rahman, T. Xing, Y. Chen, A. M. Glushenkov. J. Mater. Chem. A. 2, 4282-4291 (2014), DOI: 10.1039/c3ta14643j

37. H. Lv, S. Qiu, G. Lu, Y. Fu, X. Li, C. Hu, J.Liu. Electrochimica Acta. 151, 214-221 (2015), DOI: 10.1016/j.electacta.2014.11.013

38. G. Wang, R. Pandey, S.P. Karna. ACS Appl. Mater. Interfaces. 7 (21), 11490 - 11496 (2015), DOI: 10.1021/ acsami.5b02441

39. A.A. Ashcheulov, O.N. Manyk, T.O. Manyk, S.F. Marenkinb, V.R. Bilynskiy-Slotylo. Inorganic Materials. 49 (8), $766-769$ (2013), DOI: 10.1134/ S0020168513070017

40. M. Zhao, X. Zhang, L.Li. Scientific Reports. 5, 16108 (2015), DOI: 10.1038/srep16108 